\title{
Recurrent Cutaneous Melanoma
}

National Cancer Institute

\section{Source}

National Cancer Institute. Recurrent Cutaneous Melanoma. NCI Thesaurus. Code C9097.

Reemergence of a melanoma of the skin after a period of remission. 